\title{
Issues in implementing a scientific data grid based on peer-to-peer architecture
}

\begin{abstract}
Grid and peer-to-peer (P2P) networks enable the sharing and aggregation of geographically distributed resources. Grids mostly deal with large computational problems and provide geographically distributed resources for large-scale data-intensive applications that generate large data sets, whereas $\mathrm{P} 2 \mathrm{P}$ provides a way for sharing huge volumes of data. In a modern scientific computing, the communities of researchers involves in organizing, moving, visualizing, and analyzing massive amounts of a very large data collections in a geographically distributed environment. Research in the area of grid and P2P computing; have given us various ideas and solutions to address the problems. One of the most challenging issues in grid computing that address above requirements is locating and sharing remote data. In this paper, we illustrate the idea of using $\mathrm{P} 2 \mathrm{P}$ approach for locating and sharing data in scientific data grid environment through our P2P scientific data grid framework.
\end{abstract}

Keyword: Data grid; Data sharing; Peer-to-peer 